\title{
A Rare Case Report of Dorsal Epidural Abscess Caused by Streptococcus Agalactiae in a Diabetic Patient \\ Jihane Belkhair ${ }^{1 *}$, Arsène Ntini ${ }^{2}$, Taoufik Rokni ${ }^{1}$, Said Ait Benali ${ }^{2}$, Nabila Soraa ${ }^{1}$
}

\author{
${ }^{1}$ Laboratory of Microbiology, Errazi Hospital, Mohamed VI University Hospital, Marrakech; Faculty of Medicine and Pharmacy, Cadi Ayyad \\ University, Marrakech, Morocco \\ ${ }^{2}$ Neurosurgery Department, Errazi Hospital, Mohamed VI University Hospital, Marrakech; Faculty of Medicine and Pharmacy, Cadi Ayyad University, \\ Marrakech, Morocco
}

DOI: $10.36347 /$ simcr.2020.v08i08.013

| Received: 18.07.2020 | Accepted: 27.07.2020 | Published: 28.08.2020

*Corresponding author: Jihane Belkhair

\section{Abstract}

Background: The infectious epidural abscess by Streptococcus agalactiae is a rare, and a surgical emergency with a reserved prognosis. We report, to our knowledge, a rare case of S. agalactiae epiduritis. Case report: This observation reports the case of a diabetic patient who presented with compressive neurological signs. The biological assessment showed an inflammatory syndrome, and a magnetic resonance imaging objectified epiduritis. The bacteriological study of curettage biopsy made it possible to isolate a $S$. agalactiae. The evolution was favorable under antibiotic treatment with disappearance of neurological and infectious signs. Conclusion: Epidural abscess with S. agalactiae must be evoked with a cytobacteriological study in front of a suggestive symptomatology, especially in case of favoring factor.

Keywords: Epiduritis, Streptococcus agalactiae, bacteriological study, diabetes.

Copyright @ 2020: This is an open-access article distributed under the terms of the Creative Commons Attribution license which permits unrestricted use, distribution, and reproduction in any medium for non-commercial use (NonCommercial, or CC-BY-NC) provided the original author and source are credited.

\section{INTRODUCTION}

Epidural abscess is a rare and better known by the development of neurosurgery, but extremely serious condition, with a potential life threat especially in patients with predisposing factor such as diabetes, hemodialysis, cancer, HIV, hematological diseases [1]. Therapeutic management must be rapid and effective to avoid serious neurological sequelae. It is a pathology whose diagnosis is clinico-radiological. Magnetic resonance imaging is the means of positive and etiological diagnosis. The etiology is most often due to Staphylococcus aureus, rarely to a Streptococcus agalactiae $[1,2]$.

The urgency of diagnosing and treating is explained by the serious condition that can affect the functional prognosis and the using an appropriate antibiotic to stop antibiotic-resistant strains of microorganisms [2]. Despite the medical progress, the delays before diagnosis are very variable because of the often-deceptive clinical signs, and the taking of this disease remains poorly codified. The objective of this report was to highlight the rare presence of $S$. agalactiae, in a case of epiduritis of a diabetic patient, who presented with compressive neurological signs in a febrile context.

\section{CASE RePORT}

A 62 year old woman, having had type 2 diabetes mellitus for 12 years, on oral antidiabetic drugs (metformin), who presented to the emergency room with the complaint of pain localized at the spinal radicular level, motor and sensory disorders of the lower limbs, sphincter disorders aggressive installation; associated with weakness, fevers and chills that had a gradual onset. All moving for a month.

\section{Examination}

On nervous system examination founded a spinal compression syndrome; motor and sensory disorders of the lower limbs, and sphincter disorders. The rest of the clinical examination was unremarkable.

\section{Radiological findings}

Magnetic resonance imaging(MRA)showed a T1 hypointense and a T2 hypersignal well as a thickening of spinal epidural of D1 to D5(Figure 1).

\section{Lab findings}

Complete blood count reports showed with leukocytosis at $12 \mathrm{G} / \mathrm{L}$ predominantly neutrophilic polynuclear at $9 \mathrm{G} / \mathrm{L}$, with normal hemoglobin and platelet count. Blood biochemistry showed C-reactive protein (CRP) at $179 \mathrm{mg} / \mathrm{l}$. For cytobacteriological 
study of the biopsy curettage, the direct examination after Gram staining showed the presence of Grampositive Cocci in chains, the culture allowed isolating beta hemolytic colonies on blood agar (figure 2). The latex agglutination test objectified group B streptococci. The identification of the species isolated $S$. agalactiae by the BD Phoenix ${ }^{\mathrm{TM}}$ automated and the antibiogram has been done according to the recommendation of EUCAST-SF 2019 on chocolate agar. S. agalactiae was multi-sensitive to beta-lactam, glycopeptide, macrolide (erythromycin, spiramycin, lincomycin), fluoroquinolones (moxifloxacin, levofloxacin), gentamycin 500 and trimethoprim / sulfamethoxazole. Blood cultures were negative.

\section{Intervention and management}

After a posterior approach, general anesthesia, the patient underwent decompressive laminectomy, evacuation of abscess, and debridement at the level of D1 to L1 with curettage biopsy. The curettage product was sent for a bacteriological study.

The evolution under treatment with clavulanic acid amoxicillin and gentamicin for two months was marked by the disappearance of neurological signs and clinic-biological infectious signs.

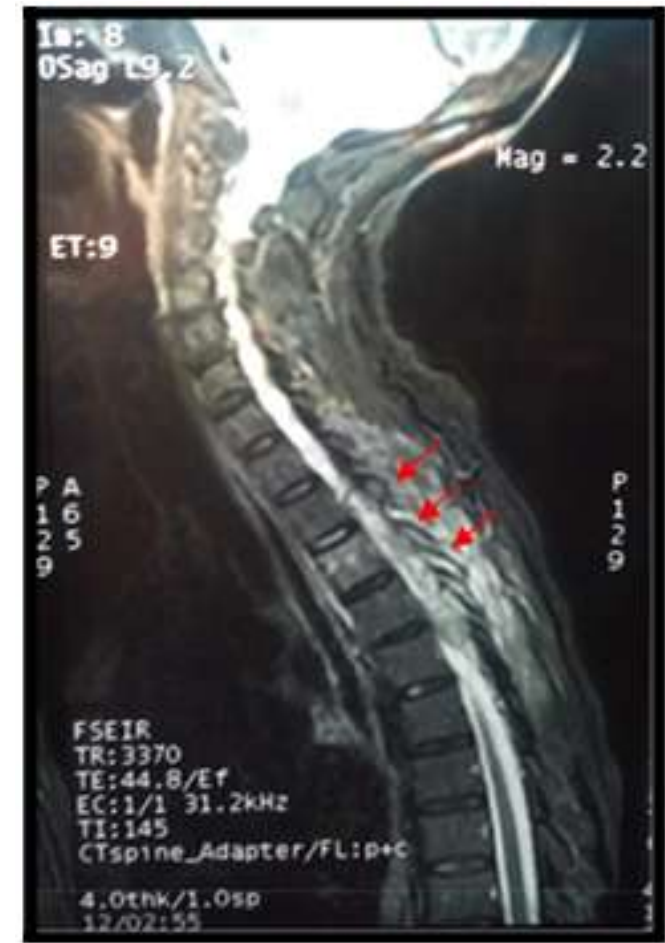

Fig-1: Sagittal plane of thoracic spine Magnetic resonance imaging show an epidural abscess at the level of D1 to D5 (arrows)

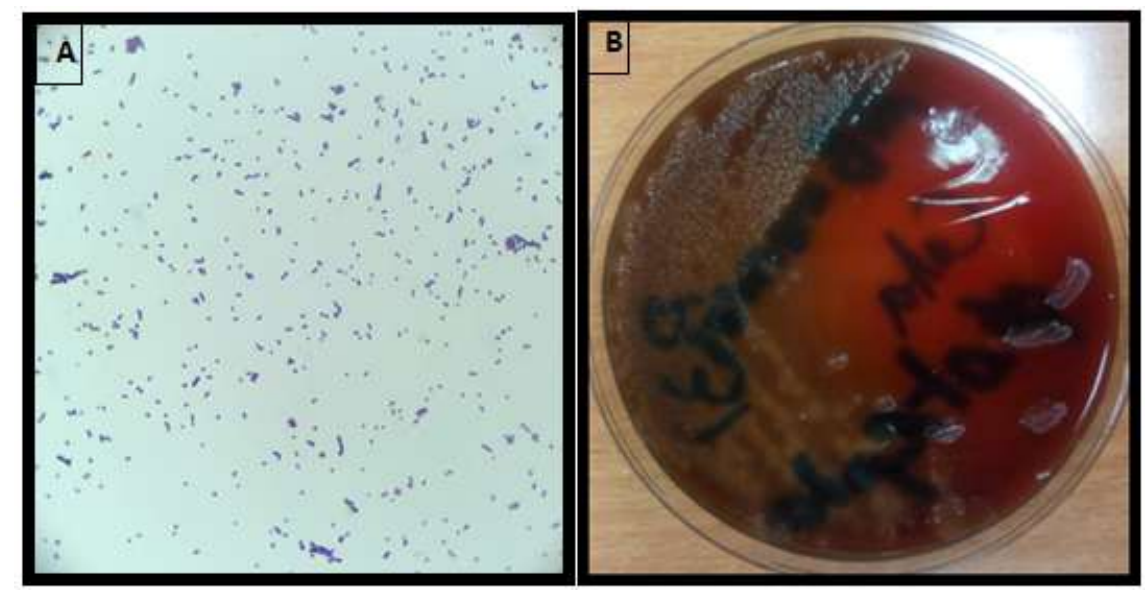

Fig-2: A: Gram positive cocci after Gram staining B: Hemolytic beta-streptococcus colonies on blood agar

\section{DiSCUSSION}

S. agalactiae (Group B Streptococcus) is a Gram-positive capsulated bacterium, commensal to the digestive and vaginal tracts, remains a continued source of morbidity and mortality in high-risk populations [3]. $S$. agalactiae infections, apart from maternofetal infections, are mainly characterized by bacteremia and arthritis, endocarditis and meningitis [4] with an increasing tendency of mortality of GBS infection in elderly patients. Epidural abscess (EA) is favored by an underlying terrain: diabetes, alcoholism, renal failure, hematologic malignancies, cancer, and iatrogenic causes such as acupuncture, traumatism or corticoid epidural injection $[4,5]$.
Epidural abscess is an infection of bacterial origin by accumulation of pus from the epidural space which is mechanically responsible for spinal cord compression [6]. Epidural abscess is an emergency diagnostic and surgical therapeutic by decompression, pus drainage, and antibiotic therapy which cover gram negative bacteria, gram positive bacteria, and anaerobes to to minimize the neurological deficit $[6,7]$.

EA is estimated at 1 case per $20 \quad 000$ hospitalizations, with a peak incidence between 55 and 65 years, with a sex-ratio of 1 [8], which occurs on a favorable site of type diabetes, immunocompromised field, chronic ethylic [9]. This patient was 62 years old; diabetes was the favoring factor. The main symptom of epiduritis is spinal pain in more than $70 \%$ of cases [10]. Onset of symptoms begins with spinal pain, spinal 
stiffness, and/or fever. The combination of pain or stiffness in the neck and fever should raise suspicion of EA, which is the classic triad of spinal EA $[6,9,10]$. All these signs were present in this patient. The diagnosis of certainty is radiological based on MRI by showing a hyperintensity in $\mathrm{T} 2$ with epidural enhancement, with a sensitivity and specificity that exceeds $90 \%$ [10]. The thoracic location is the most described, and the involvement affects the posterior epidural compartment in two-thirds. The patient had a dorsal localization of 4 vertebral stages (D1 to D5), and the front door was not known.

Routine biological examinations point to an infectious etiology in the presence of nonspecific neurological signs, with an infectious syndrome; polynuclear leukocytosis, and elevation of CRP. More than $60 \%$ of patients with EA have had leukocytosis [6]. Assessment of Serum C-reactive protein (CRP) [6, 7]. These parameters were used in this patient to evaluate the effectiveness of the treatment put in place.

The search in Pub Med by tapping on the search bar "epidural abscess" (on 17/09/2019), was found 2890 publications including 15 references were reported infectious origin to s. agalactiae of epiduritis. Epiduritis with $S$. agalactiae is rarely described. $S$. aureus is the incriminated germ in $75 \%$ of cases [6].

Treatment usually requires a combination of a drainage procedure and antibiotic therapy, like most abscesses. Medical management includes a polyantibiotic treatment prolonged from one to three months [6]. They should choose antibiotics for Staphylococcus $s p$, Streptococcus sp, Hemophilus sp, and anaerobes, and they can then change treatment based on cultures and sensitivities. The patient was put on amoxicillin clavulanic acid and gentamicin. The indications for surgery are spinal cord compression and / or the absence of a favorable course of antibiotics alone [6]. The prognosis is variable; the evolution can be favorable if the care is early [8].

\section{CONCLUSION}

Clinicians must evaluate all patients who experience acute or progressive back pain, fever, and local spine tenderness for the possibility of the EA. Bacteriological examination remains essential to confirm the infectious origin of epiduritis. It must be carried out systematically to isolate the bacterium responsible and study its sensitivity to antibiotics.

\section{ACKNOWLEDGEMENTS}

The authors would like to thank all laboratory technicians who have been involved in the identification of the species isolated and the antibiogram, and all equip of professors and doctors of Neurosurgery Department who participated and helped in the treatment of the patient.

\section{Author Contributions}

JB, AN, and TR: write the manuscript, and collect information and researchers. NS and ABS supervise and correct the final version of the manuscript.

\section{REFERENCES}

1. Six A., Joubrel C, Tazi A, Poyart C. Infections materno-foetales à Streptococcus agalactiae. Presse Med. 2014; 43 (6P1) : 706-714.

2. Lefebvre N, Forestier E, Mohseni-Zadeh M, Remy $\mathrm{V}$, Lesens $\mathrm{O}$, Kuhnert $\mathrm{C}$. Les infections invasives à Streptococcus agalactiae chez l'adulte. Medmal. 2007;37(12): 796-801.

3. Kang T, Park SY, Lee SH, Park JH, Suh SW. Spinal epidural abscess successfully treated with biportal endoscopic spinal surgery. Medicine. 2019 Dec;98(50).

4. Darouiche RO. Spinal epidural abscess. N Engl J Med. 2006; 355(19): 2012-20.

5. Huang PY, Chen SF, Chang WN, Lu CH, Chuang YC, Tsai NW, Chang CC, Wang HC, Chien CC, Chen SH, Huang CR. Spinal epidural abscess in adults caused by Staphylococcus aureus: clinical characteristics and prognostic factors. Clinical neurology and neurosurgery. $2012 \mathrm{Jul}$ 1;114(6):572-6.

6. Avilucea FR, Alpesh AP. Epidural infection: Is it really an abscess? Surg Neurol Int. 2012; 3(Suppl 5): S370-S376.

7. Soehle M, Wallenfang T. Spinal epidural abscesses: Clinical manifestations, prognostic factors, and outcomes. Neurosurgery. 2002;51:79.

8. Tompkins M, Panuncialman I, Lucas P, Palumbo M. Spinal epidural abscess. Journal of Emergency Medicine. 2010;39(3):384-390.

9. Sendi P, Bregenzer T, Zimmerli W. Spinal epidural abscess in clinical practice. Quarterly Journal of Medicine. 2008;101(1):1-12.

10. Zhang JH, Wang ZL, Wan L. Cervical epidural analgesia complicated by epidural abscess: A case report and literature review. Medicine. 2017 Oct;96(40). 